\title{
Mash1 and Math3 Are Required for Development of Branchiomotor Neurons and Maintenance of Neural Progenitors
}

\author{
Ryosuke Ohsawa, Toshiyuki Ohtsuka, and Ryoichiro Kageyama \\ Institute for Virus Research, Kyoto University, Kyoto 606-8507, Japan
}

Basic helix-loop- helix (bHLH) transcription factors are known to play important roles in neuronal determination and differentiation. However, their exact roles in neural development still remain to be determined because of the functional redundancy. Here, we examined the roles of neural bHLH genes Mash1 and Math3 in the development of trigeminal and facial branchiomotor neurons, which derive from rhombomeres 2- 4. In Math3-null mutant mice, facial branchiomotor neurons are misspecified, and both trigeminal and facial branchiomotor neurons adopt abnormal migratory pathways. In Mash1;Math3 double-mutant mice, trigeminal and facial branchiomotor neurons are severely reduced in number partly because of increased apoptosis. In addition, neurons with migratory defects are intermingled over the midline from either side of the neural tube. Furthermore, oligodendrocyte progenitors of rhombomere 4 are reduced in number. In the absence of Mash1 and Math3, expression of Notch signaling components is severely downregulated in rhombomere 4 and neural progenitors are not properly maintained, which may lead to intermingling of neurons and a decrease in oligodendrocyte progenitors. These results indicate that Mash1 and Math3 not only promote branchiomotor neuron development but also regulate the subsequent oligodendrocyte development and the cytoarchitecture by maintaining neural progenitors through Notch signaling.

Key words: bHLH; branchiomotor neuron; facial nucleus; hindbrain; Notch signaling; trigeminal nucleus

\section{Introduction}

During vertebrate neural development, progenitors undergo proliferation and differentiation into neurons and glial cells, and these steps are regulated by multiple basic helix-loop-helix (bHLH) genes such as Mash1 and Neurogenin2 (for review, see Kageyama and Nakanishi, 1997; Lee, 1997; Bertrand et al., 2002; Ross et al., 2003). Mash1 is expressed in the ventral telencephalon and regulates formation of GABAergic interneurons, whereas Neurogenin2 is expressed in the dorsal telencephalon and regulates formation of glutamatergic pyramidal neurons (Casarosa et al., 1999; Horton et al., 1999; Fode et al., 2000; Parras et al., 2002; Schuurmans et al., 2004). In the developing retina, Mash1 regulates bipolar cell development, whereas another bHLH gene, NeuroD, promotes amacrine cell genesis (Morrow et al., 1999; Hatakeyama et al., 2001; Inoue et al., 2002). Thus, distinct bHLH genes regulate distinct neuronal subtype specification. However, bHLH genes alone are not sufficient for generation of the neuronal diversity, but other types of transcription factor genes such as homeodomain genes are required. In the retina, the homeodo-

Received Nov. 10, 2004; revised April 11, 2005; accepted May 6, 2005.

This work was supported by the Sasagawa Research Grant from the Japan Scientific Society and research grants from the Ministry of Education, Culture, Sports, Science, and Technology of Japan. R.0. was supported by The 21st Century Center of Excellence Program of the Ministry of Education, Culture, Sports, Science, and Technology of Japan. We thank Dr. François Guillemot for Mash1-null mice and discussion. The monoclonal antibodies to Islet1 and Nkx2.2 were obtained from the Developmental Studies Hybridoma Bank (University of lowa, lowa City, IA).

Correspondence should be addressed to Dr. Ryoichiro Kageyama, Institute for Virus Research, Kyoto University, Shogoin-Kawahara, Sakyo-ku, Kyoto 606-8507, Japan. E-mail: rkageyam@virus.kyoto-u.ac.jp.

DOI:10.1523/JNEUROSCI.4621-04.2005

Copyright $\odot 2005$ Society for Neuroscience $\quad$ 0270-6474/05/255857-09\$15.00/0 main gene Chx10 regulates the layer identity, whereas Mash1 determines the neuronal fate of the Chx10-specified layer (Hatakeyama et al., 2001).

Generation of the hindbrain neurons is also controlled by combinations of bHLH and homeodomain genes. The embryonic hindbrain is composed of reiterated segmental structures called rhombomeres. From each rhombomere, specific types of neurons are generated. For example, trigeminal branchiomotor neurons derive from rhombomere 2 (r2) and $\mathrm{r} 3$, whereas facial branchiomotor neurons derive from r4 (Marshall et al., 1992; Auclair et al., 1996; McKay et al., 1997; for review, see Chandrasekhar, 2004). These neurons migrate to the dorsolateral positions of the hindbrain. Recent studies revealed that Mash1 regulates neurogenesis of the ventral $\mathrm{r} 4$ in combination with the homeodomain gene Phox $2 b$ (Dubreuil et al., 2002; Pattyn et al., 2004). In the absence of either Mash 1 or Phox $2 b$, neurons are still generated, although they are misspecified and reduced in number in Phox2b-null embryos (Hirsch et al., 1998; Pattyn et al., 2000, 2003b), whereas in Mash1;Phox2b double-null embryos, neurons are completely missing (Pattyn et al., 2004). These results indicate that Mash 1 and Phox $2 b$ cooperatively promote neurogenesis in r4. However, it remains to be determined whether the homeodomain gene Phox $2 b$ alone can rescue all aspects of loss of Mash 1 or whether as yet unidentified bHLH genes may participate in the neurogenesis process. In the absence of Phox $2 b$, expression of another bHLH gene, Math3, is lost (Pattyn et al., 2000), whereas misexpression of Phox $2 b$ induces Math 3 expression (Dubreuil et al., 2000), raising the possibility that Math3 could compensate Mash1 for some aspects of development of hindbrain neurons. 
Here, we found that Mash1 and Math3 are transiently coexpressed by branchiomotor neuron progenitors. Furthermore, development of branchiomotor neurons is more severely affected in Mash1;Math3 double-mutant embryos than in Mash1-null and Math3-null embryos. Our results indicate that Mash1 and Math3 cooperatively regulate development of trigeminal and facial branchiomotor neurons and maintenance of neural progenitors by activation of Notch signaling. Furthermore, in the absence of Mash1 and Math3, oligodendrocyte development is affected because neural progenitors are not properly maintained.

\section{Materials and Methods}

Mouse strains. All animals used in this study were maintained and handled according to protocols approved by Kyoto University. Generation of Mash1 and Math3 mutant mice was reported previously (Guillemot et al., 1993; Tomita et al., 2000). Genotyping of mutants was performed by PCR. The primer sequences for genotyping of the Mash1 mutant allele were as follows: 5'-ACGACTTGAACTCTATGGCGGGTTCTC-3', 5'-GCCACTCTCAGGGGCCAAGACTGAAGTTAA-3', and 5'-AAATTAAGGGCCAGCTCATTCCTCCACTCA-3'. PCR products were loaded in a $1.5 \%$ agarose gel for electrophoresis. A $350 \mathrm{bp}$ band indicates a wild-type allele, and a $280 \mathrm{bp}$ band indicates a mutant allele. For genotyping of the Math3 mutant allele, the primer sequences were 5 '-GTATATGAAATCCAAGGACATGGTGGAGCT-3'， 5'-TGACTCTTCGAGCCCTGAATCTTTCAAGGC-3', and 5'-CGCTGATCAGCCTCGACTGTGCCTTCTAGT-3'. A 260 bp band indicates a wild-type allele, and a $350 \mathrm{bp}$ band indicates a mutant allele.

Immunohistochemistry. Embryos were fixed in $4 \%$ paraformaldehyde at $4^{\circ} \mathrm{C}$ for $3 \mathrm{~h}$, washed in ice-cold PBS three times, equilibrated in $20 \%$ sucrose at $4^{\circ} \mathrm{C}$, embedded in OCT compound, and frozen at $-80^{\circ} \mathrm{C}$. The sections were made by cryostat (Leica, Nussloch, Germany) at 16 $\mu \mathrm{m}$ thickness and incubated in 5\% normal goat serum and $0.1 \%$ Triton X-100 at room temperature for $1 \mathrm{~h}$, then incubated with primary antibodies against microtubule-associated protein 2 (MAP2; 1:1000; Sigma, St. Louis, MO), Nkx2.2 (1:200; 74.5A5; Hybridoma Bank, Iowa City, IA), Ki-67 (1:5000; BD PharMingen, San Diego, CA), N-cadherin (1:500; Transduction Laboratories, Lexington, KY), and Islet1 (1:200; 40.2; Hybridoma Bank). A biotinylated antibody against mouse IgG (1:200; Vector Laboratories, Burlingame, CA) was used for a secondary antibody. FITC-avidinD (1:1000; Vector Laboratories) was added to detect the signal.

In situ hybridization. In situ hybridization on tissue sections was performed as described previously (Hirata et al., 2001), using Mash1, Math3, Hes5, Delta-like1, Delta-like3, Islet1, Sim1, c-Ret, Phox 2b, Ebf-1, Sonic hedgehog $(S h h)$, and neo probes. RNA probes were labeled with digoxigenin. Embryos were dissected and fixed in $4 \%$ paraformaldehyde at $4^{\circ} \mathrm{C}$ overnight, rinsed with ice-cold PBS three times, equilibrated in $20 \%$ sucrose, embedded in OCT compound, and frozen at $-80^{\circ} \mathrm{C}$. Tissue sections were made by cryostat. The sections were treated with proteinase $\mathrm{K}$, refixed with $0.2 \%$ glutaraldehyde and $4 \%$ paraformaldehyde, rinsed with $0.1 \%$ Tween 20 in PBS, and hybrid-

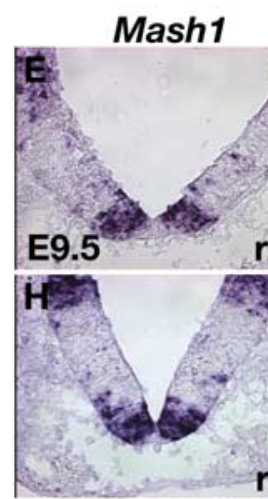

ized with RNA probe in $50 \%$ formamide, $5 \times$ SSC, $1 \%$ SDS, $50 \mu \mathrm{g} / \mathrm{ml}$ heparin, and $50 \mu \mathrm{g} / \mathrm{ml}$ tRNA solution at $65^{\circ} \mathrm{C}$ overnight. The sections were washed in $50 \%$ formamide, $5 \times$ SSC, and $1 \%$ SDS at $65^{\circ} \mathrm{C}$, treated with ribonuclease, washed in $50 \%$ formamide and $2 \times$ SSC, washed in Tris-buffered saline, and incubated with alkaline phosphataseconjugated antibody against digoxigenin at $4^{\circ} \mathrm{C}$ overnight. After incubation, the sections were washed with $0.1 \%$ Tween 20 in Tris-buffered saline three times, then in $100 \mathrm{~mm} \mathrm{NaCl}, 100 \mathrm{~mm}$ Tris- $\mathrm{HCl}, \mathrm{pH}$ 9.5, 50 $\mathrm{mm} \mathrm{MgCl}_{2}$, and $0.1 \%$ Tween 20 solution once. For a color development reaction, 4-nitroblue tetrazolium chloride and 5-bromo-4-chloro-3indolyl phosphate (BCIP) were used as substrates.

For double RNA in situ hybridization, Mash1 and Math3 RNA probes were labeled with FITC and digoxigenin, respectively. Detection of Math 3 mRNA was performed as described above. After the first color development reaction, the sections were washed in Tris-buffered saline three times and incubated at $65^{\circ} \mathrm{C}$ to eliminate the alkaline phosphatase
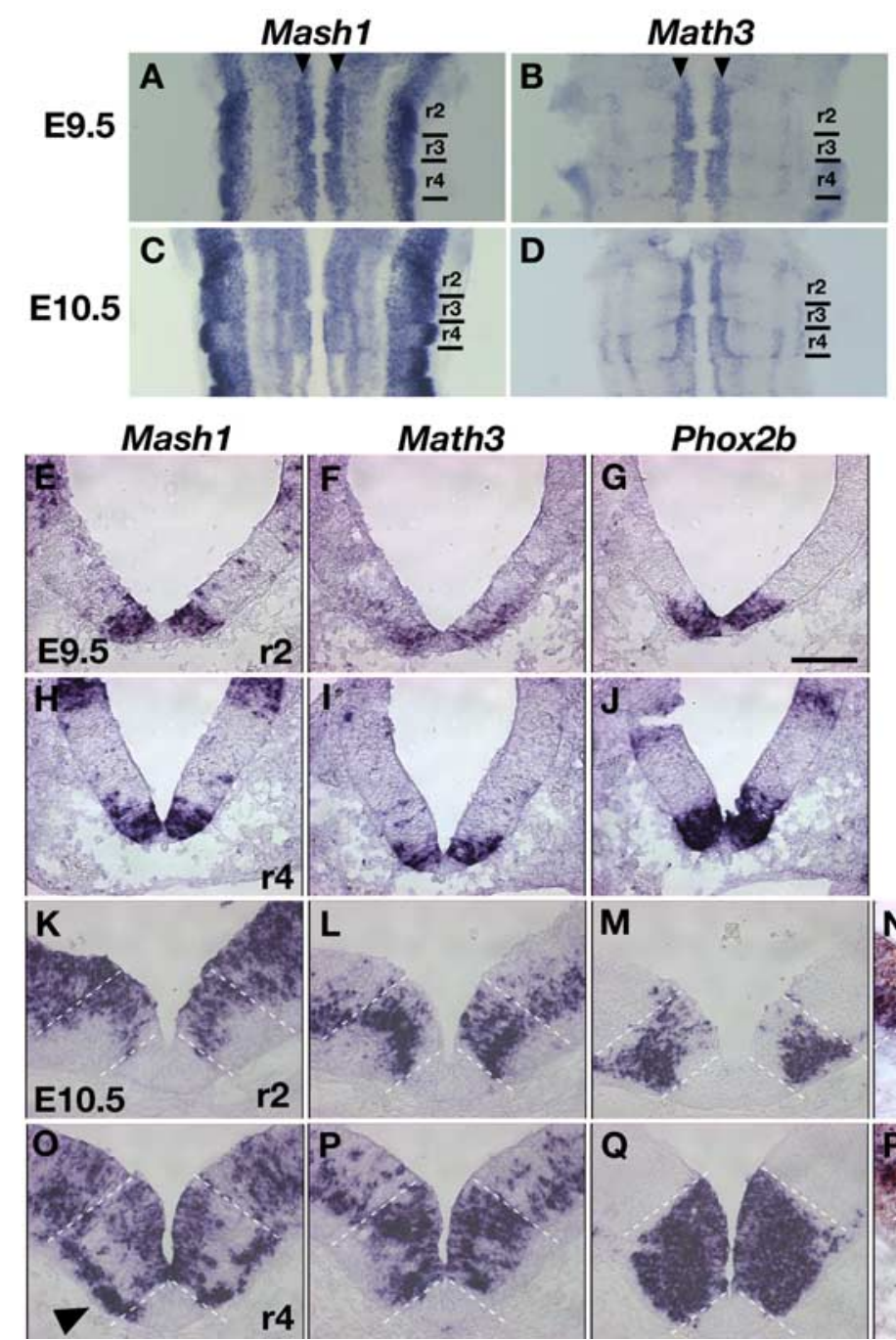

\section{Mash1 Math3}

Figure 1. Expression patterns of Mash1 and Math3 in the developing hindbrain. In situ hybridization was performed for Mash 1 $(A, C, E, H, K, \mathbf{O}, N, R)$, Math $3(B, D, F, I, L, P, N, R)$, and $\operatorname{Phox2b}(G, J, M, Q) . A-D$, The hindbrain was cut along the roof plate and flat mounted. At E9.5 and E10.5, Mash1 was strongly expressed in the ventral hindbrain where trigeminal and facial branchiomotor neurons arise $(\boldsymbol{A}$, arrowheads, $\boldsymbol{C}$. Math 3 was also expressed in the ventral hindbrain at $\mathrm{E} 9.5$ and $\mathrm{E} 10.5$ ( $\boldsymbol{B}$, arrowheads, $\boldsymbol{D})$. $\boldsymbol{E}-\boldsymbol{R}$, In situ hybridization was performed with transverse sections through $\mathrm{r} 2(\boldsymbol{E}-\mathbf{G}, \boldsymbol{K}-\boldsymbol{N})$ and $\mathrm{r} 4(\boldsymbol{H}-\boldsymbol{J}, \mathbf{0}-\boldsymbol{R})$ at $\mathrm{E9.5}(\boldsymbol{E}-\boldsymbol{J})$ and $\mathrm{E} 10.5(\boldsymbol{K}-\boldsymbol{R})$. In $\mathrm{r} 2$ and $\mathrm{r} 4$, which give rise to trigeminal and facial branchiomotor neurons, respectively, Mash 1 was expressed by subsets of ventricular cells as well as by cells migrating out of the ventricular zone $(\boldsymbol{E}, \boldsymbol{H}$, between dashed lines in $K, \boldsymbol{O})$. Mash 1 was also expressed in postmitotic inner-ear efferent neurons ( $\boldsymbol{O}$, arrowhead), as reported previously (Tiverson et al., 2003). Math3 was expressed by cells in the ventricular zone as well as in the outer layers of $\mathrm{r} 2$ and $r 4(\boldsymbol{F}, \boldsymbol{I}$, between dashed lines in $\boldsymbol{L}, \boldsymbol{P})$. Phox $2 \boldsymbol{b}$ expression was well overlapped with Mash1 and Math3 expression domains in both $r 2$ and $r 4(G, J, M, Q)$. At least some of the ventricular and migrating cells coexpressed Mash1 and Math3 ( $\boldsymbol{N}, \boldsymbol{R}$, between dashed lines). Scale bar, $100 \mu \mathrm{m}$. 


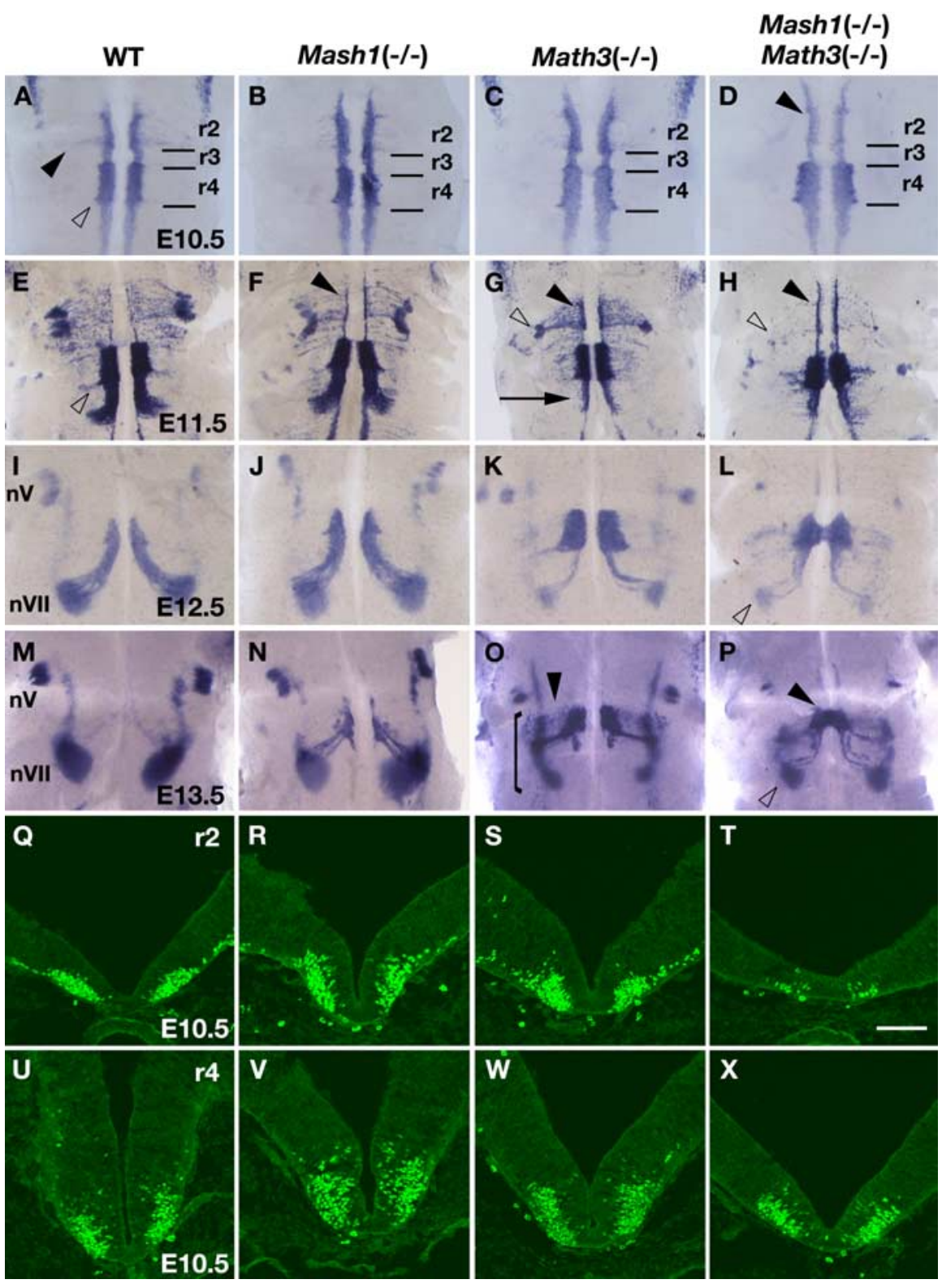

Figure 2. Developmental defects of trigeminal and facial branchiomotor neurons in mutant embryos. $\boldsymbol{A}-\boldsymbol{P}$, In situ hybridization for Islet1 was performed with the wild-type $(\boldsymbol{A}, \boldsymbol{E}, \boldsymbol{I}, \boldsymbol{M}), \operatorname{Mash} 1(-/-)(\boldsymbol{B}, \boldsymbol{F}, \boldsymbol{J}, \mathbf{N}), \operatorname{Math} 3(-/-)(\boldsymbol{C}, \mathbf{G}, \boldsymbol{K}, \mathbf{O})$, and Mash1(-/-); Math3(-/-) $(\boldsymbol{D}, \boldsymbol{H}, \boldsymbol{L}, \boldsymbol{P})$ hindbrain. The samples were cut along the roof plate and flat mounted. $\boldsymbol{A}-\boldsymbol{D}$, At $\mathrm{E} 10.5$, trigeminal $(\boldsymbol{A}$, filled arrowhead) and facial $\left(\boldsymbol{A}\right.$, open arrowhead) branchiomotor neurons $\left(I /\right.$ let $\left.1^{+}\right)$were born in the ventral region of $\mathrm{r} 2-\mathrm{r} 4$ and started migration in the wild type. Similarly, these neurons were born in Mash1(-I-) (B) and Math3(-I-) (C). In Mash1(-/-); Math3 $(-/-)$, fewer neurons were born from $\mathrm{r} 2(\boldsymbol{D}$, arrowhead) than the others $(\boldsymbol{A}-\boldsymbol{C}) . \boldsymbol{E}-\boldsymbol{H}$, AtE11.5, the majority of trigeminal branchiomotor neurons migrated to the dorsolateral position of $\mathrm{r} 2$ in the wild type and $\operatorname{Mash} 1(-/-)(\boldsymbol{E}, \boldsymbol{F})$, although some neurons remained in the ventral region in Mash $1(-I-)(\boldsymbol{F}$, arrowhead). Many of facial branchiomotor neurons migrated from $r 4$ into $r 5$ and $r 6$ in the wild type $(\boldsymbol{E}$, open arrowhead) and Mash $1(-/-)(\boldsymbol{F})$. In Math3 $(-/-)$, many neurons still remained in $\mathrm{r} 2$ (G, filled arrowhead), resulting in a small nucleus ( $\mathbf{G}$, open arrowhead). Only subsets of $\mathrm{r} 4$ neurons migrated into $\mathrm{r} 5(\mathbf{G}$, arrow). In Mash1 $(-/-)$;Math3 $(-/-)$, most of the 2 neurons did not migrate $(\boldsymbol{H}$, filled arrowhead), resulting in a very small trigeminal nucleus ( $\boldsymbol{H}$, open arrowhead). Only subsets of $\mathrm{r} 4$ neurons migrated into $r 5$. $\boldsymbol{I}-\boldsymbol{L}$, AtE12.5, most trigeminal branchiomotor neurons finished migration, forming the trigeminal nucleus $(\mathrm{nV})$ in the wild type and Mash $1(-/-)(I, J)$. Many of the facial branchiomotor neurons migrated into the dorsal region of $\mathrm{r} 6$, forming the facial nucleus (nVII) in the wild type and Mash $1(-/-)(I, J)$. In contrast, most facial branchiomotor neurons still remained in $\mathrm{r} 4$ in Math3 $(-/-)(\boldsymbol{K})$ and Mash1 $(-/-)$;Math3( $(-/-)$, resulting in a very small facial nucleus ( $K, L$, open arrowhead). In Mash $1(-/-)$;Math $3(-/-), r 4$ neurons were intermingled across the midline ( $\boldsymbol{L})$. $\boldsymbol{M}-\boldsymbol{P}$, At E13.5, most facial branchiomotor neurons finished migration, forming the facial nucleus (nVII) in the wild type and Mash $1(-/-)(\boldsymbol{M}, \boldsymbol{N})$. In contrast, in Math3(-I-), some facial branchiomotor neurons migrated within $\mathrm{r} 4$, forming an elongated nucleus ( 0 , arrowhead and bracket). In Mash1(-/-);Math3(-/-), still many $\mathrm{r} 4$ neurons remained in the ventral $r 4$, resulting in a small nucleus ( $\boldsymbol{P}$, open arrowhead). More neurons were intermingled across the midline ( $\boldsymbol{P}$, filled arrowhead). $\mathbf{Q}-\boldsymbol{X}$, Immunohistochemistry against Islet1 was performed with transverse sections of $\mathrm{r} 2(\boldsymbol{Q}-\boldsymbol{T})$ and $\mathrm{r} 4(\boldsymbol{U}-\boldsymbol{X})$ at E10.5. Islet $1^{+}$cells were significantly reduced in number in Mash1(-/-); Math $3(-/-)$ r2 $(T)$. Scale bar, $100 \mu \mathrm{m}$. WT, Wild type. activity for a primary reaction. The sections were then incubated with alkaline phosphataseconjugated antibody against FITC at $4^{\circ} \mathrm{C}$ overnight. For the Mash1 mRNA detection, 2[4-iodophenyl]-3-[4-nitrophenyl]-5-phenyltetrazolium chloride and BCIP were used as substrates.

Whole-mount in situ hybridization was performed as described previously (Bessho et al., 2001) with the following minor modification. The hindbrain was dissected and cut at the dorsal edge of the neural tube before fixation. After performing whole-mount in situ hybridization, hindbrain tissues were flat mounted on a slide glass.

Terminal deoxynucleotidyl transferasemediated dUTP nick end labeling assay. Embryos were dissected and fixed in $4 \%$ paraformaldehyde at $4^{\circ} \mathrm{C}$ for $3 \mathrm{~h}$. After fixation, the embryos were rinsed with ice-cold PBS, equilibrated in 20\% sucrose, embedded in OCT compound, and frozen at $-80^{\circ} \mathrm{C}$. The sections were made by cryostat at $16 \mu \mathrm{m}$ thickness. Terminal deoxynucleotidyl transferase-mediated dUTP nick end labeling (TUNEL) assay was performed as indicated in the protocol provided by a manufacturer (In Situ Cell Death Detection kit; Roche, Mannheim, Germany).

Bromodeoxyuridine incorporation assay. Six hundred microliters of $10 \mathrm{mg} / \mathrm{ml}$ bromodeoxyuridine (BrdU) in PBS (6 mg) were injected intraperitoneally into pregnant mice. After 30 min, embryos were dissected and fixed in $4 \%$ paraformaldehyde at $4^{\circ} \mathrm{C}$ overnight. Then embryos were rinsed with ice-cold PBS three times, equilibrated in $20 \%$ sucrose, and embedded in OCT compound. The tissue sections were rinsed with PBS three times, blocked in 5\% normal goat serum and $0.1 \%$ Triton X-100 at room temperature for $1 \mathrm{~h}$, and treated with $2 \mathrm{~N} \mathrm{HCl}$ at $37^{\circ} \mathrm{C}$ for $30 \mathrm{~min}$. After acidic treatment, the sections were neutralized in $0.1 \mathrm{M}$ sodium tetraborate, $\mathrm{pH} 8.0$, rinsed with $\mathrm{PBS}$ three times, and incubated with anti-BrdU antibody (1:1000; Sigma). Signal detection was performed as immunohistochemistry.

\section{Results}

Mash 1 and Math3 are expressed in branchiomotor neuron progenitors in the hindbrain

To characterize the roles of Mash1 and Math3 in hindbrain development, we first examined their expression patterns by in situ hybridization. The hindbrain was cut along the roof plate and flat mounted to locate the expression domains. At embryonic day 9.5 (E9.5), Mash1 was expressed mainly in two longitudinal columns. Expression in the ventral column occurred at a high level from $\mathrm{r} 2$ to $\mathrm{r} 4$, where trigeminal and facial branchiomotor neurons arise (Fig. 1A, arrowheads). This expression continued but became less significant at E10.5 (Fig. 1C). Math3 expression was also observed in the ventral column from $\mathrm{r} 2$ to $\mathrm{r} 4$ at E9.5 (Fig. $1 B$, arrowheads) and 
continued at E10.5 (Fig. 1D). In r2 and r4, Mash1 expression occurred mainly in the ventricular zone, whereas it continued in subsets of cells migrating out of the ventricular zone at both E9.5 and E10.5 (Fig. $1 E, H, K, O)$. Math3 expression also occurred in the ventricular zone, but Math 3 was expressed mainly by the cells in the outer layers of $\mathrm{r} 2$ and $\mathrm{r} 4$ during this period (Fig. $1 F, I, L, P)$. Some of these cells coexpressed Mash1 and Math3 (Fig. 1N,R). These results suggest that Mash1 and Math3 are expressed by both neural progenitors and differentiating neurons of $\mathrm{r} 2$ to $\mathrm{r} 4$.

Because the ventral region of $\mathrm{r} 2-\mathrm{r} 4$ is known to give rise to branchiomotor neurons, we next compared the expression patterns of Mash1 and Math3 with those of Phox $2 b$, which has been shown to control branchiomotor neuron generation (Pattyn et al., 1997, 2000). Phox $2 b$ expression was well overlapped with the Mash1 and Math3 expression domains in $\mathrm{r} 2$ and $\mathrm{r} 4$ (Fig. 1G,J, between dashed lines in $M, Q$ ). These results suggest that Mash1 and Math3 are expressed at distinct but overlapping stages by differentiating trigeminal and facial branchiomotor neurons and their progenitors.

\section{Defects of trigeminal branchiomotor neuron development in Math3(-/-) and Mash1(-/-);Math3(-/-) mutants}

To investigate the roles of Mash1 and Math3 in branchiomotor neuron development, we first analyzed trigeminal motor neurons in Mash1-null and/or Math3-null mice. We used Islet1 as a general motor neuron marker (Ericson et al., 1992). In wild-type embryos, trigeminal branchiomotor neurons arose from the ventral region of r2 and r3 between E9.5 and E10.5 (Taber Pierce, 1973; Marshall et al., 1992) and migrated dorsolaterally to form the trigeminal motor nucleus (Fig. 2A, arrowhead, Q) (Altman and Bayer, 1982), which extended in the dorsolateral position of $\mathrm{r} 2$ and $\mathrm{r} 3$. This migration was almost completed by E11.5, and only a few motor neurons remained in the ventral region (Fig. $2 E)$. Although trigeminal motor neurons appeared to be generated normally in Mash1-null embryos (Fig. $2 B, F, R$ ), a few more motor neurons still remained in the ventral region adjacent to the floor plate of $\mathrm{r} 2$ and $\mathrm{r} 3$ at E11.5 (Fig. $2 \mathrm{~F}$, arrowhead), suggesting that the development of trigeminal branchiomotor neurons is slightly delayed compared with the wild type. Similarly, more motor neurons remained in the ventral region of $\mathrm{r} 2$ and $\mathrm{r} 3$ at E11.5 in Math3-null embryos (Fig. 2G, filled arrowhead), although trigeminal motor neurons were normally born at E10.5 (Fig. 2C,S). In addition, the trigeminal motor nucleus of Math3null embryos was smaller than that of the wild type (Fig. $2 G$, open arrowhead). This is likely attributable to a migratory defect, because more neurons remained in the ventral region of Math 3 mutants (Fig. 2G, filled arrowhead). Furthermore, the rostral neurons migrated dorsocaudally to converge on a small caudal nucleus in r2 (Fig. 2G, open arrowhead). These results indicate that Math3 is important for migratory behavior of trigeminal branchiomotor neurons.
Because the overlapping patterns of Mash1 and Math3 expression suggest redundant roles of these bHLH genes, we next examined Mash1;Math3 double-mutant embryos. In the double mutants, trigeminal motor neurons were severely reduced in number at E10.5 and E11.5 (Fig. 2D,H, filled arrowhead, T). Very few cells migrated dorsolaterally, and, as a result, the trigeminal motor nucleus was very small at E11.5 (Fig. $2 \mathrm{H}$, open arrowhead) compared with the wild type, Mash1-null, and Math3-null embryos (Fig. $2 E-G$ ). At later stages, the trigeminal motor nucleus $(\mathrm{nV})$ was also very small (Fig. $2 L, P$ ). To further investigate the cause of the defects of the double-mutant embryos, we examined apoptotic cell death by TUNEL assay. In wild-type embryos and Math3-null embryos, no overt apoptotic cell death was observed in r2 (Fig. 3A,C), whereas a few TUNEL-positive cells were detected in Mash1-null embryos (Fig. 3B). In contrast, TUNEL-positive cells were significantly increased in r2 of Mash1; Math3 double-mutant embryos (Fig. 3D). These apoptotic cells expressed Nkx2.2 (Fig. 3I) but were negative for the neuronal marker MAP2 (Fig. $3 K$ ), suggesting that cells undergo apoptosis before becoming mature neurons in the absence of Mash1 and Math3. These results indicate that Mash1 and Math3 are required for migration and survival of immature trigeminal branchiomotor neurons.

\section{Defects of facial branchiomotor neuron development in Math3 and the double mutants}

We next examined development of facial branchiomotor neurons in mutant embryos. Facial branchiomotor neurons are known to adopt unique migratory pathways (Garel et al., 2000). In wild-type embryos, facial branchiomotor neurons arose at $\mathrm{r} 4$ from E9.5-E11.5 (Fig. 2A,E, open arrowheads, $U$ ) (McKay et al., 
1997; Pattyn et al., 2003b). After E10.5, they migrated caudally, keeping its position adjacent to the floor plate. After entering r6, they switched the direction and migrated dorsolaterally, forming the nucleus at r6 (Fig. 2I, M, nVII). This migration was almost completed at E14.5. In Mash1 mutant embryos, generation and migration of facial branchiomotor neurons seemed to be normal at E10.5-E12.5 (Fig. 2B,F,J,V). However, at E13.5, many cells still remained near the floor plate of $\mathrm{r} 4$ or on the way to r6 (Fig. $2 N$ ), although some of them in $\mathrm{r} 4$ could be aberrant inner-ear efferent neurons (Tiveron et al., 2003). These results suggest that in the absence of Mash1, facial branchiomotor neurons exhibit a delay of migration. However, at later stages, the facial motor nucleus was formed, and no overt defect was observed (data not shown). In contrast, Math3-null embryos exhibited abnormal migration of facial branchiomotor neurons. At E11.5, very few cells reached r6 (Fig. $2 G$, arrow), whereas the majority of the cells remained in the ventral region of 4 of Math3-null embryos (Fig. $2 G)$. At E12.5, very few cells reached the dorsolateral position of r6, whereas the majority still remained in the ventral region of $\mathrm{r} 4$ (Fig. $2 \mathrm{~K}$ ). At E13.5, in Math3-null embryos, some of these neurons migrated dorsolaterally within $\mathrm{r} 4$ (Fig. 2O, arrowhead), forming an elongated nucleus between $\mathrm{r} 4$ and r6 (Fig. 2O, bracket), in contrast to the wild-type facial motor nucleus, which resided in $\mathrm{r} 6$ (Fig. $2 M$ ).

In the Mash1;Math3 double-mutant hindbrain, although many neurons were born in the ventral $\mathrm{r} 4$ at E10.5 (Fig. 2D, X), very few cells reached $\mathrm{r} 6$ at E11.5, whereas the majority of the cells remained in the ventral region of $\mathrm{r} 4$ (Fig. $2 H$ ). At E12.5 and E13.5, only subsets of cells reached the dorsolateral position of $\mathrm{r} 6$ (Fig. $2 L, P$, open arrowheads), whereas other subsets migrated within $\mathrm{r} 4$, forming an elongated nucleus between $\mathrm{r} 4$ and $\mathrm{r} 6$, which is similar to but smaller than the Math3-null nucleus (Fig. 2O,P). In Mash 1; Math3 double-mutant r4, TUNEL-positive cells were increased (Fig. $3 H$ ) compared with the others (Fig. 3E-G). These apoptotic cells expressed Nkx2.2 (Fig. 3J) but were negative for the neuronal marker MAP2 (Fig. $3 L$ ), suggesting that cells undergo apoptosis before becoming mature neurons in the absence of Mash1 and Math3. These results indicate that Mash1 and Math3 are required for migration and survival of immature facial branchiomotor neurons. Strikingly, the left and right neurons, which are normally separated by the floor plate, were fused across the midline in $\mathrm{r} 4$ of the double mutants. This fusion started at E12.5 (Fig. 2L) and became more severe at E13.5 (Fig. 2 P, arrowhead). Thus, in the absence of Mash1 and Math3, the cytoarchitecture of $\mathrm{r} 4$ was disrupted.

\section{Misspecification of facial branchiomotor neurons in Math3 mutant embryos}

To characterize the migratory defects of facial branchiomotor neurons in Math3 mutant mice, we examined several markers that are expressed during migration. Expression of $c$-Ret, a gene for the glial cell line-derived neurotrophic factor receptor, initiates in facial branchiomotor neurons only after they migrate into 5 (Garel et al., 2000). In the wild-type embryos, few, if any, cells expressed $c$-Ret at E10.5 (Fig. 4A). In contrast, in Math3 mutant embryos, $c$-Ret expression occurred ectopically in $\mathrm{r} 4$ (Fig. $4 B$ ), suggesting that this ectopic expression may affect migration of branchiomotor neurons. Sim1, a gene for a bHLH-PAS (motif of proteins PER-ARNT-SIM) transcription factor expressed by V3 interneurons of the spinal cord (Fan et al., 1996; Briscoe et al., 1999), was not expressed in the wild-type hindbrain (Fig. 4C). In contrast, $\operatorname{Sim} 1$ was ectopically expressed in the ventral region of r4 of Math3(-/-) at E11.5 (data not shown) and E13.5 (Fig.
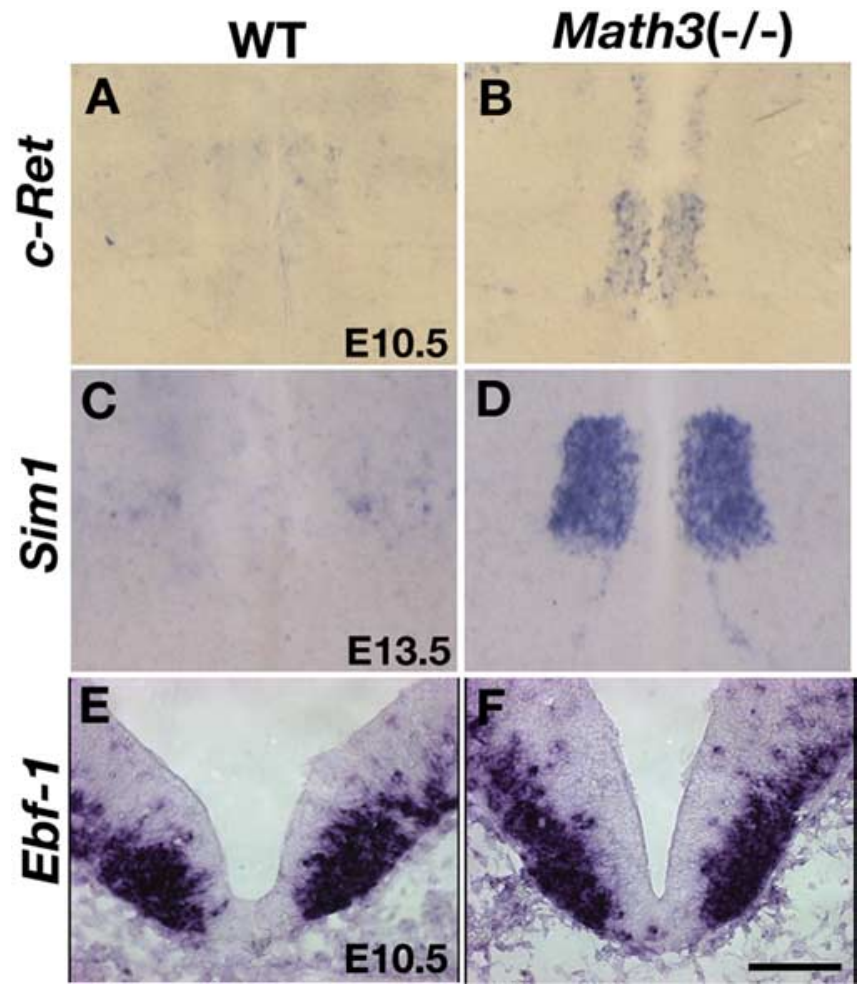

Figure 4. Ectopic gene expression in Math3(-I-) hindbrain. In situ hybridization for c-Ret $(\boldsymbol{A}, \boldsymbol{B}), \operatorname{Sim} 1(\boldsymbol{C}, \boldsymbol{D})$, and $E b f-1(\boldsymbol{E}, \boldsymbol{F})$ was performed with flat-mounted hindbrain $(\boldsymbol{A}-\boldsymbol{D})$ and transverse $(\boldsymbol{E}, \boldsymbol{F})$ sections of $\mathrm{r} 4$ at the indicated time points. $\boldsymbol{A}, \boldsymbol{B}, \boldsymbol{c}-$ Ret expression was rarely detectable in the wild type although ectopically upregulated in the ventral $r 4$ of $M a t h 3(-/-)$. C, D, Sim 1 expression was not detected in the wild type but occurred at a high level in the ventral r4 of Math $3(-/-)$. Thus, branchiomotor neurons of $\mathrm{r} 4$ were misspecified in Math3(-/-).E, $\boldsymbol{F}$, Ebf-1, which is required for migration of branchiomotor neurons, was not significantly affected in Math 3 mutant embryos, suggesting that Ebf- 1 is not involved in the migratory defects of Math3 mutant facial branchiomotor neurons. Scale bar: (in $\boldsymbol{F}) \boldsymbol{E}, \boldsymbol{F}, 100 \mu \mathrm{m}$. WT, Wild type.

$4 D)$. These results indicate that facial branchiomotor neurons are misspecified in Math3 mutant embryos, raising the possibility that this misspecification may lead to the migratory defect of these neurons.

In contrast, expression of the bHLH gene Ebf-1, which is required for correct migration of branchiomotor neurons (Garel et al., 2000), was not significantly affected in Math3 mutant embryos (Fig. $4 E, F$ ). Thus, these Math3-null cells are not totally transformed into other cell types, although they are severely misspecified.

\section{Loss of neural progenitors in the ventral region of Mash1; Math3 double-mutant r4}

In the absence of Mash1 and Math3, facial branchiomotor neurons on either side were fused across the midline in $\mathrm{r} 4$. To examine this fusion, we made transverse sections through $\mathrm{r} 4$ at E13.5. In the wild type, the inner surface was covered by the MAP2negative ventricular zone, which contains neural progenitors (Fig. $5 A$ ). In contrast, in the ventral region of the double-mutant $\mathrm{r} 4$, the ventricular cells were missing and instead MAP2-positive neurons from either side of the neural tube were fused together over the floor plate (Fig. 5B, boxed region). Furthermore, these MAP2-positive neurons were exposed directly to the fourth ventricle (Fig. $5 B^{\prime}$ ). Neural progenitors are known to have the tight junction and adherens junction at the apical side and thereby prevent neurons from migrating into the ventricular lumen: premature loss of neural progenitors leads to intermingling of neu- 
rons from the left and right walls of the neural tube (Hatakeyama et al., 2004). In the wild type, the adherens junction molecule $\mathrm{N}$-cadherin was highly expressed at the apical surface (Fig. 5C), whereas it was missing in the double mutants (Fig. 5D, arrowheads), suggesting that neural progenitors are prematurely lost. In agreement with this observation, BrdU uptake and expression of Ki-67, a marker for mitotic cells, were reduced in the double mutants compared with the wild type as early as E10.5 (Fig. 5E-H), indicating that dividing neural progenitors are not properly maintained in the absence of Mash1 and Math3. The floor plate, which expresses $\mathrm{Shh}$, was maintained in the double mutants as in the wild type at both E10.5 and E13.5 (Fig. 5I-L), indicating that the midline structure is not lost in the double mutants. Thus, inactivation of Mash1 and Math3 is likely to lead to premature loss of neural progenitors and their apical junctional complex and thereby allows facial branchiomotor neurons to intermingle each other from either side of the neural tube. This observation implies that Mash1 and Math3 are required not only for normal development of branchiomotor neurons but also for maintenance of neural progenitors and the cytoarchitecture.

\section{Notch signaling is impaired in Mash1(-/-);Math3(-/-) \\ double-mutant mice}

Because Notch signaling is known to regulate maintenance of neural progenitors (Ohtsuka et al., 1999; Gaiano et al., 2000; Hitoshi et al., 2002), we next addressed whether Notch signaling is affected in the double-mutant embryos. We first examined expression of Hes5, a downstream Notch effector (Ohtsuka et al., 1999). In the ventral $\mathrm{r} 4$ of the wild-type, Mash1(-/-), and Math3(-/-) embryos, expression of Hes5 was observed at E10.5 (Fig. 6A-C). In contrast, in the double-mutant $\mathrm{r} 4$, Hes 5 expression was significantly downregulated (Fig. $6 D$, between dashed lines). At E11.5, Hes 5 expression remained at a very low level in the double mutants, whereas Hes5 expression was still maintained in the wildtype, Mash1-null, and Math3-null r4 (Fig. 6E-H), although it was also slightly downregulated in Mash1-null r4 (Fig. 6F). These results indicate that Notch signaling is affected in the doublemutant embryos, which accounts for loss of neural progenitors.

Because neuronal bHLH genes are known to activate expression of Notch ligands such as Delta, we next examined expression of Delta-like1 (Dll1) and Dll3. In the wild-type and Math3(-/-) embryos, Dll1 expression occurred at E10.5 and E11.5 in the ventral domain where facial motor neurons are generated, whereas it was slightly decreased in Mash1(-/-) embryos (Fig. $6 I-K, M-O)$. In contrast, in the double mutants, Dll1 expression in the ventral $\mathrm{r} 4$ was downregulated at E10.5 (Fig. $6 \mathrm{~L}$ ) and mostly missing at E11.5 (Fig. 6 P). Similarly, expression of Dll3 was missing in the double mutants at E10.5 (Fig. 6T, between dashed lines), in contrast to the wild-type, Mash1-null, and Math3-null embryos (Fig. 6Q-S). Thus, expression of the Notch ligands is also lost in the absence of Mash1 and Math3, which is likely to lead to downregulation of Hes5 in neighboring cells.

The loss of Notch ligand expression could be attributable to either downregulation of the gene expression in cells that should normally express Mash1/Math3 or apoptotic loss of these cells. To differentiate between these possibilities, we monitored Mash1/Math3-expressing cells in the double-mutant hindbrain. In Mash1 and Math3 mutant alleles, a neomycin-resistant (neo) gene cassette was inserted into each locus in the same and reverse orientations, respectively (Guillemot et al., 1993; Tomita et al., 2000). Thus, we could use the neo antisense and sense strand probes as an indicator for the Mash1 and Math3 promoter activities, respectively. In the wild-type embryos, Math3 was expressed by cells adjacent to the floor plate of $\mathrm{r} 4$ at E11.5 (Fig. 7A, arrow). In the double-mutant embryos, the antisense neo was expressed in the same domain (Fig. 7B, arrow). Similarly, the sense neo was expressed in the same domain (Fig. 7D). Thus, the cells that should normally express Mash1/Math3 did not die but still ex- 


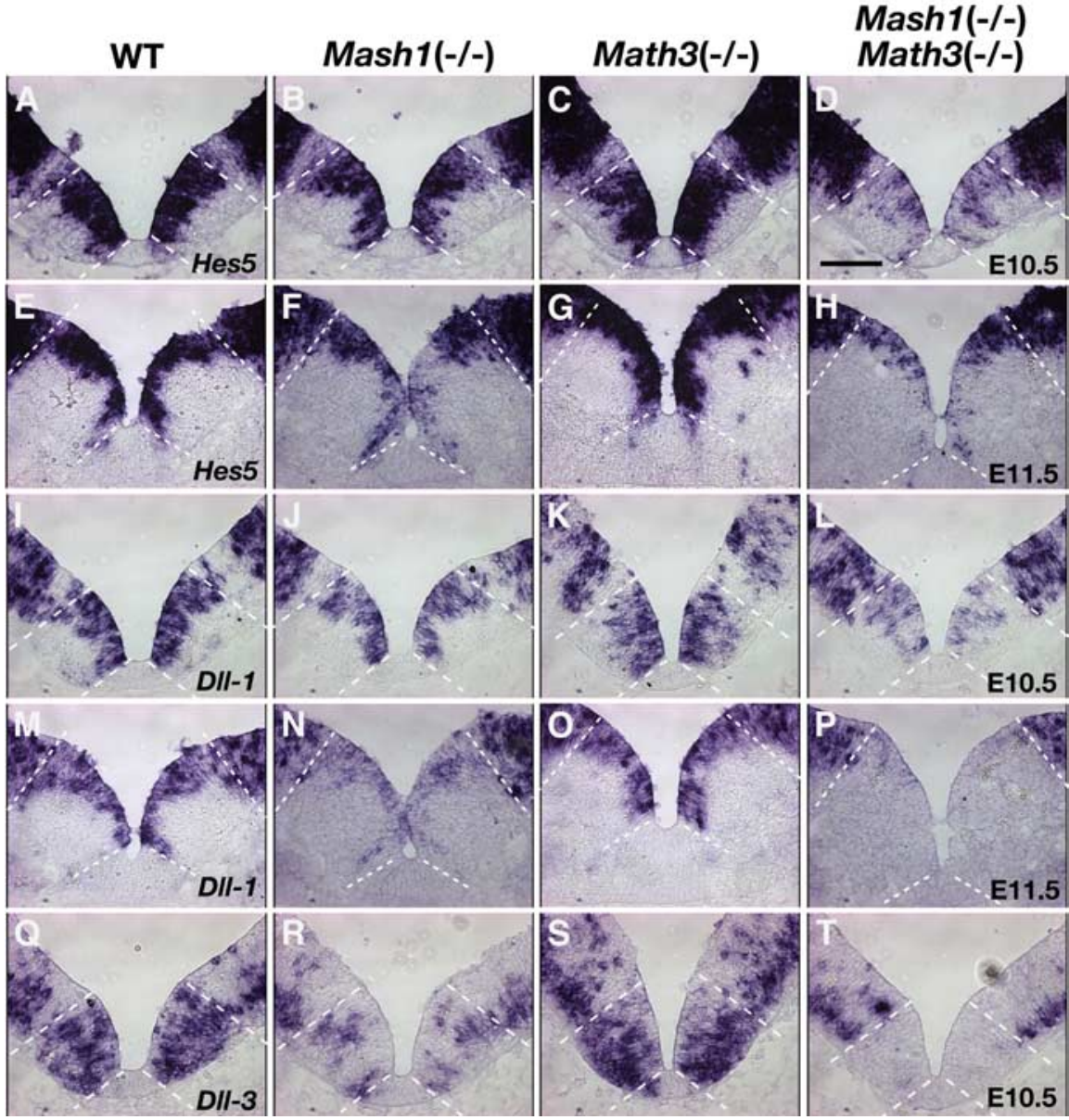

Figure 6. Impairment of Notch signaling in the ventral $\mathrm{r} 4$ of Mash1(-/-);Math3(-/-). In situ hybridization for Hes5 $(\boldsymbol{A}-\boldsymbol{H})$, DII1 (I-P), and DII3 (Q- $\boldsymbol{T})$ was performed with transverse sections of the ventral $\mathrm{r} 4$ at E10.5 $(\boldsymbol{A}-\boldsymbol{D}, \boldsymbol{I}-\mathbf{L}, \mathbf{Q}-\boldsymbol{T})$ and E11.5 $(\boldsymbol{E}-\boldsymbol{H}, \boldsymbol{M}-\boldsymbol{P})$. In Mash1(-/-);Math3(-/-), expression of Hes5 $(\boldsymbol{D}, \boldsymbol{H}), \operatorname{Dll1}(\boldsymbol{L}, \boldsymbol{P})$, and DII3 $(\boldsymbol{T})$ was significantly downregulated in the ventral $r 4$, which gives rise to facial branchiomotor neurons (between dashed lines). In Mash $1(-/-)$, expression of Hes5 $(\boldsymbol{B}, \boldsymbol{F}), \mathrm{D} \| 11(\boldsymbol{J}, \boldsymbol{N})$, and D\|I $(\boldsymbol{R})$ was slightly downregulated compared with the wild type and Math3(-/-). Scale bar, $100 \mu \mathrm{m}$. WT, Wild type.

isted in the double-mutant embryos at E11.5, indicating that loss of Dll1 and Dll3 expression is attributable to downregulation of the gene expression rather than to cell death.

We also noted that antisense neo-positive cells were present in a higher density in the double-mutant ventricular zone than in the wild type, which exhibited a salt-and-pepper pattern (Fig. 7, compare arrows in $A, B$ ). This is probably because Notch signaling, which represses neuronal bHLH gene expression of neighboring cells in a way known as "lateral inhibition," is impaired in the double mutants.

\section{The delay of oligodendrocyte development in Mash1(-/-); Math3(-/-) mouse embryos}

The ventral region is known to give rise to oligodendrocytes after generating motor neurons. Because neural progenitors were significantly decreased in the double mutants, we next examined the development of oligodendrocytes in the ventral $r 4$ of the double mutants. In r4, expression of the early oligodendrocyte marker PDGFR $\alpha$ was observed at E12.5 in the wild-type, Mash1(-/-) and Math3(-/-) embryos (Fig. $8 A-C$ ). In contrast, in Mash1(-/-);Math3(-/-) embryos, PDGFR $\alpha$-positive cells were severely reduced in number (Fig. $8 D$ ). These results suggest that impairment of maintenance of neural progenitors may lead to reduction of oligodendrocyte progenitors. At E14.5, however, PDGFR $\alpha$-positive cells were found to be distributed throughout the hindbrain in the double-mutant embryos (data not shown). Thus, development of oligodendrocytes is not dependent on Mash1 or Math3, and the initial decrease of oligodendrocyte progenitors can be recovered at later stages.

\section{Discussion}

Mash 1 and Math3 cooperatively regulate development of trigeminal and facial branchiomotor neurons

We found here that Mash1 and Math3 cooperatively regulate development of trigeminal and facial branchiomotor neurons. In the absence of Mash1 and Math3, these neurons exhibit migratory defects and apoptosis, resulting in formation of very small trigeminal and facial nuclei. Furthermore, in r4, neurons with migratory defects are intermingled over the midline from either side of the neural tube, and oligodendrocyte progenitors are reduced in number. Thus, Mash1 and Math3 not only promote branchiomotor neuron development but also regulate the subsequent oligodendrocyte development and the cytoarchitecture in the hindbrain. It has been shown that neurons derived from the ventral $\mathrm{r} 4$ are completely missing in Mash1;Phox $2 b$ double-null embryos, whereas they are still generated in Mash1null and Phox $2 b$-null embryos, indicating that Mash 1 and Phox $2 b$ cooperatively promote neurogenesis in the ventral r4 (Pattyn et al., 2004). However, because Phox $2 b$ upregulates Math3 expression (Dubreuil et al., 2000, 2002; Pattyn et al., 2000), our present data raise the possibility that at least some aspects of Phox $2 b$ functions in rescuing Mash 1 activities are mediated by Math3. Particularly, apoptosis and decrease of expression of Notch signaling molecules observed in Mash1null mice are more severely affected in Mash1;Math3 double-null mice, indicating that Math3 may compensate Mash1 for cell survival and activation of Notch signaling.

\section{Mash1 and Math3 function at a neuronal differentiation process in the hindbrain}

Our present study established the roles of Mash1 and Math3 in the differentiation process of cranial branchiomotor neurons. In the double mutants, although trigeminal and facial branchiomotor neurons seemed to be born normally, they underwent cell death at E10.5. As a result, expression of the neuronal markers MAP2 and $\beta$-tubulin III exhibited marked reduction (data not shown). Furthermore, the majority of the surviving neurons did not properly migrate but remained in the ventral region, where they were born. Thus, in the absence of Mash1 and Math3, the neuronal fate seems to be determined, but the subsequent differentiation process is impaired, although Mash1 and Math3 are known to regulate the neuronal fate determination process. It is possible that other bHLH genes may compensate for the deter- 


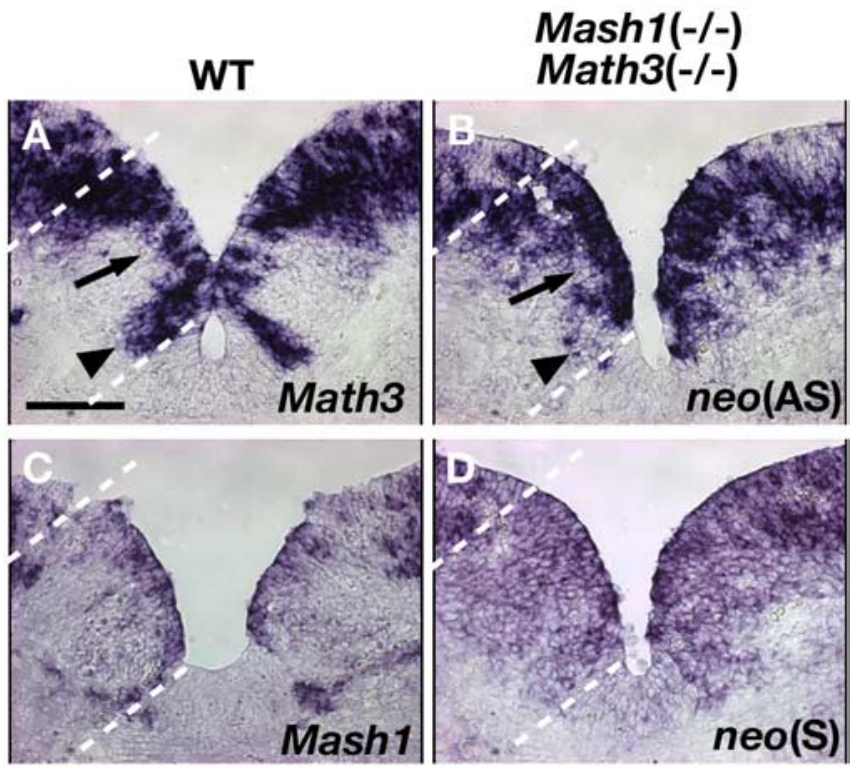

Figure 7. The cells that should normally express Mash1 and Math3 remained in Mash1(-/-); Math $3(-/-)$. In situ hybridization was performed with transverse sections of the ventral $\mathrm{r} 4$ at E11.5. A, Math3 was expressed in a salt-and-pepper pattern in the ventral $r 4$ of the wild type (arrow). B, Expression of the antisense neomycin mRNA under the control of the Math3 promoter was detected in the ventral $\mathrm{r} 4$ of Mash $1(-/-)$; Math3 $(-/-)$ (between dashed lines). C, Mash1 was expressed in the ventral $r 4$ of the wild type. $D$, Expression of the sense neomycin mRNA under the control of the Mash 1 promoter was detected in the ventral $\mathrm{r} 4$ of Mash $1(-/-)$; Math3(-/-) (between dashed lines). Thus, the cells that should normally express Mash1/ Math 3 remained in the double mutants. Cells adjacent to the floor plate were decreased in the double mutants (compare arrowheads in $\boldsymbol{A}, \boldsymbol{B}$ ). Scale bar, $100 \mu \mathrm{m}$. WT, Wild type.
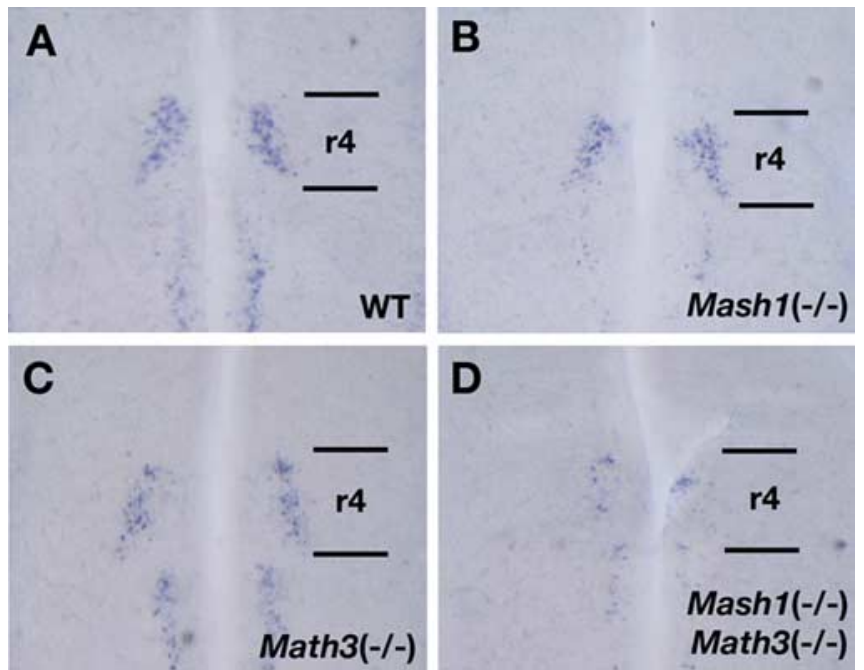

Figure 8. Impairment of oligodendrocyte development in the ventral $\mathrm{r} 4$ of Mash1(-/-); Math3(-/-). In situ hybridization for PDGFR $\alpha$ was performed at E12.5. PDGFR $\alpha$ expression was significantly downregulated in the ventral $r 4$ of Mash1(-/-); Math3(-/-) (D) compared with the others $(\boldsymbol{A}-\boldsymbol{C})$. Thus, oligodendrocyte progenitors were significantly reduced in number in the double mutants. WT, Wild type.

mination step. Alternatively, Phox $2 b$ itself has neuronal fate determination activities, as described previously (Dubreuil et al., 2000; Pattyn et al., 2004), although it remains to be determined whether Phox $2 b$ can directly activate the neuronal fate determination program without inducing neuronal bHLH gene expression.
Mash1 and Math3 regulate Notch ligand expression in $\mathrm{r} 4$ It was reported previously that Dll1 expression is completely lost in $\mathrm{r} 4$ of Phox $2 b$;Mash1 double-mutant embryos. This loss of Dll1 expression in Phox $2 b$; Mash 1 double mutants is probably as a result of the complete blockade of neurogenesis (Pattyn et al., 2004). In the present study, we showed that Dll1 and Dll3 expression is also lost in the ventral $\mathrm{r} 4$ of the Mash1;Math3 double mutants, although many neurons are born. It is thus likely that Mash1 and Math3 cooperatively maintain Notch ligand expression in these neurons and thereby activate Notch signaling and downregulate Mash1 and Math3 expression in neighboring cells, resulting in a salt-and-pepper expression pattern. Consistent with this idea, in the absence of Mash1 and Math3, expression of the Notch effector Hes5 is lost, and the Mash1 and Math3 promoter activities are uniformly upregulated in the double-mutant ventricular cells. Our results thus suggest that Math3, rather than Phox $2 b$, directly regulates Notch ligand expression in collaboration with Mash1.

\section{Control of facial branchiomotor neuron migration}

Facial branchiomotor neurons adopt a unique migratory pathway, and we found that in the absence of Math3, this migration is severely affected. Thus far, it has been shown that facial branchiomotor neuron progenitors and their progeny start expression of the three transcription factor genes Nkx6.1, Math3, and Ebf-1 in this order, and mutant analyses revealed that each gene is required for the normal migration. In the absence of the earliest onset gene $N k x 6.1$, facial branchiomotor neurons migrate dorsolaterally within $\mathrm{r} 4$, forming the nucleus mainly in $\mathrm{r} 4$ (Müller et al., 2003; Pattyn et al., 2003a), whereas in the absence of the latest onset gene Ebf-1, dorsolateral migration occurs in r5-r6, forming the nucleus in r5-r6 (Garel et al., 2000). In the absence of the intermediate onset gene Math3, some populations of facial branchiomotor neurons exhibit aberrant migration in $\mathrm{r} 4-\mathrm{r} 5$, whereas others exhibit normal migration within $\mathrm{r} 6$, forming an elongated nucleus extending from $\mathrm{r} 4$ to r6. Thus, inactivation of $N k x 6.1$, $M a t h 3$, and $E b f-1$ allows migration of branchiomotor neurons in $\mathrm{r} 4, \mathrm{r} 4-\mathrm{r} 6$, and r5-r6, respectively, in contrast to the wild type allowing dorsal migration only in r6. The onset of aberrant migration correlates well with the onset of gene expression, indicating that each gene prevents ectopic migration in a stage-specific manner. It is thus likely that the behavior of facial branchiomotor neurons should be controlled strictly by Nkx6.1, Math3, and $E b f-1$ in this order and, in their absence, these neurons could prematurely undergo dorsolateral migration.

c-Ret is prematurely expressed by Math3-null branchiomotor neurons in $\mathrm{r} 4$, as observed in Ebf-1 mutants, and this ectopic $c$-Ret expression probably accounts for the aberrant dorsal migration of these neurons. Although Mash1 and Math3 are functionally redundant in the branchiomotor neuron development as shown above, most of the migratory defects are attributable only to Math3 mutation, indicating that Math3 has a unique role in migration of branchiomotor neurons.

\section{Maintenance of neural progenitors by Mash1 and Math3}

We showed that oligodendrocyte progenitors are reduced in number at E12.5 in Mash1;Math3 double mutants, although the number of oligodendrocytes becomes comparable with that of the wild-type embryos at E14.5. This recovery is probably because of the highly proliferative property of oligodendrocyte progenitors, as shown in the analysis of Pax6 mutant mice (Sun et al., 1998). The reduction of oligodendrocyte progenitors at E12.5 in the double mutants is likely attributable to decrease of neural 
progenitors because Notch signaling is impaired. Another interesting observation is intermingling of branchiomotor neurons across the midline in the double mutants. This defect is also likely attributable to loss of neural progenitors. Because neural progenitors have the tight and adherens junctions at the apical side to prevent neurons from scattering, premature loss of neural progenitors allows neurons to escape into the lumen (Hatakeyama et al., 2004). Thus, Mash1 and Math3 play an important role in maintenance of neural progenitors to ensure not only a sufficient supply of glial progenitors but also the structural integrity of the nervous system.

\section{References}

Altman J, Bayer SA (1982) Development of the cranial nerve ganglia and related nuclei in the rat. Adv Anat Embryol Cell Biol 74:1-90.

Auclair F, Valdes N, Marchand R (1996) Rhombomere-specific origin of branchial and visceral motoneurons of the facial nerve in the rat embryo. J Comp Neurol 369:451-461.

Bertrand N, Castro DS, Guillemot F (2002) Proneural genes and the specification of neural cell types. Nat Rev Neurosci 3:517-530.

Bessho Y, Sakata R, Komatsu S, Shiota K, Yamada S, Kageyama R (2001) Dynamic expression and essential functions of Hes7 in somite segmentation. Genes Dev 15:2642-2647.

Briscoe J, Sussel L, Serup P, Hartigan-O’Connor D, Jessell TM, Rubenstein JL, Ericson J (1999) Homeobox gene Nkx2.2 and specification of neuronal identity by graded Sonic hedgehog signalling. Nature 398:622-627.

Casarosa S, Fode C, Guillemot F (1999) Mash1 regulates neurogenesis in the ventral telencephalon. Development 126:525-534.

Chandrasekhar A (2004) Turning heads: development of vertebrate branchiomotor neurons. Dev Dyn 229:143-161.

Dubreuil V, Hirsch MR, Pattyn A, Brunet JF, Goridis C (2000) The Phox2b transcription factor coordinately regulates neuronal cell cycle exit and identity. Development 127:5191-5201.

Dubreuil V, Hirsch MR, Jouve C, Brunet JF, Goridis C (2002) The role of Phox $2 \mathrm{~b}$ in synchronizing pan-neuronal and type-specific aspects of neurogenesis. Development 129:5241-5253.

Ericson J, Thor S, Edlund T, Jessell TM, Yamada T (1992) Early stages of motor neuron differentiation revealed by expression of homeobox gene Islet-1. Science 256:1555-1560.

Fan CM, Kuwana E, Bulfone A, Fletcher CF, Copeland NG, Jenkins NA, Crew S, Martinez S, Puelles L, Rubenstein JLR, Tessier-Lavigne M (1996) Expression patterns of two murine homologs of Drosophila single-minded suggest possible roles in embryonic patterning and in the pathogenesis of Down syndrome. Mol Cell Neurosci 7:1-16.

Fode C, Ma Q, Casarosa S, Ang SL, Anderson DJ, Guillemot F (2000) A role for neural determination genes in specifying the dorsoventral identity of telencephalic neurons. Genes Dev 14:67-80.

Gaiano N, Nye JS, Fishell G (2000) Radial glial identity is promoted by Notch1 signaling in the murine forebrain. Neuron 26:395-404.

Garel S, Garcia-Dominguez M, Charnay P (2000) Control of the migratory pathway of facial branchiomotor neurones. Development 127:5297-5307.

Guillemot F, Lo LC, Johnson JE, Auerbach A, Anderson DJ, Joyner AL (1993) Mammalian achaete-scute homolog 1 is required for the early development of olfactory and autonomic neurons. Cell 75:463-476.

Hatakeyama J, Tomita K, Inoue T, Kageyama R (2001) Roles of homeobox and bHLH genes in specification of a retinal cell type. Development 128:1313-1322.

Hatakeyama J, Bessho Y, Katoh K, Ookawara S, Fujioka M, Guillemot F, Kageyama R (2004) Hes genes regulate size, shape and histogenesis of the nervous system by control of the timing of neural stem cell differentiation. Development 131:5539-5550.

Hirata H, Tomita K, Bessho Y, Kageyama R (2001) Hes1 and Hes3 regulate maintenance of the isthmic organizer and development of the mid/hindbrain. EMBO J 20:4454-4466.

Hirsch MR, Tiveron MC, Guillemot F, Brunet JF, Goridis C (1998) Control of noradrenergic differentiation and Phox2a expression by MASH1 in the central and peripheral nervous system. Development 125:599-608.
Hitoshi S, Alexson T, Tropepe V, Donoviel D, Elia AJ, Nye JS, Conlon RA, Mak TW, Bernstein A, van der Kooy D (2002) Notch pathway molecules are essential for the maintenance, but not the generation, of mammalian neural stem cells. Genes Dev 16:846-858.

Horton S, Meredith A, Richardson JA, Johnson JE (1999) Correct coordination of neuronal differentiation events in ventral forebrain requires the bHLH factor MASH1. Mol Cell Neurosci 14:355-369.

Inoue T, Hojo M, Bessho Y, Tano Y, Lee JE, Kageyama R (2002) Math3 and NeuroD regulate amacrine cell fate specification in the retina. Development 129:831-842.

Kageyama R, Nakanishi S (1997) Helix-loop-helix factors in growth and differentiation of the vertebrate nervous system. Curr Opin Genet Dev 7:659-665.

Lee JE (1997) Basic helix-loop-helix genes in neural development. Curr Opin Neurobiol 7:13-20.

Marshall H, Nonchev S, Sham MH, Muchamore I, Lumsden A, Krumlauf R (1992) Retinoic acid alters hindbrain Hox code and induces transformation of rhombomeres 2/3 into a 4/5 identity. Nature 360:737-741.

McKay IJ, Lewis J, Lumsden A (1997) Organization and development of facial motor neurons in the kreisler mutant mouse. Eur J Neurosci 9:1499-1506.

Morrow EM, Furukawa T, Lee JE, Cepko CL (1999) NeuroD regulates multiple functions in the developing neural retina in rodent. Development 126:23-36.

Müller M, Jabs N, Lorke DE, Fritzsch B, Sander M (2003) Nkx6.1 controls migration and axon pathfinding of cranial branchio-motoneurons. Development 130:5815-5826.

Ohtsuka T, Ishibashi M, Gradwohl G, Nakanishi S, Guillemot F, Kageyama R (1999) Hes1 and Hes5 as notch effectors in mammalian neuronal differentiation. EMBO J 18:2196-2207.

Parras CM, Schuurmans C, Scardigli R, Kim J, Anderson DJ, Guillemot F (2002) Divergent functions of the proneural genes Mash1 and Ngn2 in the specification of neuronal subtype identity. Genes Dev 16:324-338.

Pattyn A, Morin X, Cremer H, Goridis C, Brunet JF (1997) Expression and interactions of the two closely related homeobox genes Phox2a and Phox 2b during neurogenesis. Development 124:4065-4075.

Pattyn A, Hirsch M, Goridis C, Brunet JF (2000) Control of hindbrain motor neuron differentiation by the homeobox gene Phox $2 \mathrm{~b}$. Development 127:1349-1358.

Pattyn A, Vallstedt A, Dias JM, Sander M, Ericson J (2003a) Complementary roles for Nkx6 and Nkx2 class proteins in the establishment of motoneuron identity in the hindbrain. Development 130:4149-4159.

Pattyn A, Vallstedt A, Dias JM, Samad OA, Krumlauf R, Rijli FM, Brunet JF, Ericson J (2003b) Coordinated temporal and spatial control of motor neuron and serotonergic neuron generation from a common pool of CNS progenitors. Genes Dev 17:729-737.

Pattyn A, Simplicio N, van Doorninck JH, Goridis C, Guillemot F, Brunet JF (2004) Ascl1/Mash1 is required for the development of central serotonergic neurons. Nat Neurosci 7:589-595.

Ross SE, Greenberg ME, Stiles CD (2003) Basic helix-loop-helix factors in cortical development. Neuron 39:13-25.

Schuurmans C, Armant O, Nieto M, Stenman JM, Britz O, Klenin N, Brown C, Langevin LM, Seibt J, Tang H, Cunningham JM, Dyck R, Walsh C, Campbell K, Polleux F, Guillemot F (2004) Sequential phases of cortical specification involve Neurogenin-dependent and -independent pathways. EMBO J 23:2892-2902.

Sun T, Pringle NP, Hardy AP, Richardson WD, Smith HK (1998) Pax6 influences the time and site of origin of glial precursors in the ventral neural tube. Mol Cell Neurosci 12:228-239.

Taber Pierce E (1973) Time and origin of neurons and in the brain stem of the mouse. Prog Brain Res 40:53-65.

Tiveron MC, Pattyn A, Hirsch MR, Brunet JF (2003) Role of Phox2b and Mash1 in the generation of the vestibular efferent nucleus. Dev Biol 260:46-57.

Tomita K, Moriyoshi K, Nakanishi S, Guillemot F, Kageyama R (2000) Mammalian achaete-scute and atonal homologs regulate neuronal versus glial fate determination in the central nervous system. EMBO J 19:54605472 . 